ARTICLE

Received 5 May 2015 | Accepted 1 Jun 2016 | Published 6 Jul 2016

DOI: $10.1038 /$ ncomms12119

OPEN

\title{
The Arabidopsis Golgi-localized GDP-L-fucose transporter is required for plant development
}

\author{
Carsten Rautengarten ${ }^{1,2, \star}$, Berit Ebert ${ }^{1,2,3, \star}$, Lifeng Liu ${ }^{4}$, Solomon Stonebloom ${ }^{1}$, Andreia M. Smith-Moritz ${ }^{1}$ \\ Markus Pauly ${ }^{4}$, Ariel Orellana 5,6 , Henrik Vibe Scheller ${ }^{1,4} \&$ Joshua L. Heazlewood ${ }^{1,2}$
}

Nucleotide sugar transport across Golgi membranes is essential for the luminal biosynthesis of glycan structures. Here we identify GDP-fucose transporter 1 (GFT1), an Arabidopsis nucleotide sugar transporter that translocates GDP-L-fucose into the Golgi lumen. Using proteo-liposome-based transport assays, we show that GFT preferentially transports GDP-L-fucose over other nucleotide sugars in vitro, while GFT1-silenced plants are almost devoid of L-fucose in cell wall-derived xyloglucan and rhamnogalacturonan II. Furthermore, these lines display reduced L-fucose content in $\mathrm{N}$-glycan structures accompanied by severe developmental growth defects. We conclude that GFT1 is the major nucleotide sugar transporter for import of GDP-L-fucose into the Golgi and is required for proper plant growth and development.

\footnotetext{
${ }^{1}$ Joint BioEnergy Institute, Physical Biosciences Division, Lawrence Berkeley National Laboratory, Berkeley, California 94702, USA. ${ }^{2}$ ARC Centre of Excellence in Plant Cell Walls, School of BioSciences, The University of Melbourne, Melbourne, VIC 3010, Australia. ${ }^{3}$ Department of Plant and Environmental Sciences, Faculty of Science, University of Copenhagen, Copenhagen DK-1871, Denmark. ${ }^{4}$ Department of Plant and Microbial Biology, University of California, Berkeley, California 94720, USA. ${ }^{5}$ Centro de Biotecnología Vegetal, Facultad de Ciencias Biológicas, Universidad Andrés Bello, Santiago RM 8370146, Chile.

${ }^{6}$ Fondo de Areas Prioritarias Center for Genome Regulation, Santiago RM 8370146, Chile. * These authors contributed equally to this study. Correspondence and requests for materials should be addressed to J.L.H. (email: jheazlewood@unimelb.edu.au).
} 
T he initial stages of the plant secretory system include the endoplasmic reticulum (ER) and the Golgi apparatus. These compartments are essential for the biosynthesis of non-cellulosic cell wall polysaccharides, and the glycosylation of proteins and lipids. These processes are mediated by glycosyltransferases that require activated substrates such as nucleotide sugars. While most nucleotide sugars are linked to uridine-5'-diphosphate (UDP), some important nucleotide sugars are linked to guanosine- $5^{\prime}$-diphosphate (GDP). Plants contain a number of GDP sugars including GDP-D-mannose (GDP-Man), GDP-L-fucose (GDP-Fuc), GDP-D-glucose (GDP-Glc) and GDP-L-galactose (GDP-Gal) ${ }^{1}$. GDP-Man is the substrate for the mannosylation of glycosylinositolphosphoceramides (GIPCs) ${ }^{2}$, dolichol- $\mathrm{P}^{3}, N$-linked glycans ${ }^{4}$ and together with GDP-Glc, important for the biosynthesis of the cell wall polymer glucomannan ${ }^{5}$. GDP-Fuc is essential for the fucosylation of xyloglucan ${ }^{6}, N$-linked glycans ${ }^{7}$ and arabinogalactan proteins ${ }^{8}$, while both GDP-Fuc and GDP-Gal are substrates for the biosynthesis of the pectic polymer rhamnogalacturonan II $(\mathrm{RG}-\mathrm{II})^{9}$.

In contrast to most nucleotide sugars, which are generated by sequential interconversions from UDP-Glc, GDP-Fuc is derived from GDP-Man. GDP-Fuc formation requires the combined activities of the GDP-D-mannose-4,6-dehydratase (GMD) and the GDP-4-keto-6-deoxy-D-mannose-3,5-epimerase-4-reductase ${ }^{10}$. A mutation in the MURUS 1 (MUR1) gene, which encodes an isoform of GMD ${ }^{11}$, results in plants with cell walls almost devoid of $\alpha$-L-fucosyl residues that are partially replaced by $\alpha$-L-galactosyl residues in xyloglucan ${ }^{12,13}$ and glycoproteins ${ }^{14}$. The mur1 mutation also affects the cell wall polymer RG-II, resulting in truncations to sidechain $\mathrm{A}^{9}$ and sometimes L-fucosyl residues substituted with L-galactosyl residues ${ }^{15}$. The mur1 mutants exhibit a severe dwarf phenotype with rosette leaves more brittle compared with wild-type plants. A second Arabidopsis gene also involved in the fucosylation of a cell wall polymer was later identified and named MURUS2 (MUR2) ${ }^{6}$. Mutants have a reduction in total cell wall $\mathrm{L}$-fucose content, which is $\sim 50 \%$ of that found in wild-type plants. MUR2 is involved in fucosylation of xyloglucan and the L-fucose content in that particular polymer is reduced to $\sim 1 \%$ in the mur 2 background compared to wildtype plants. MUR2 encodes a Golgi-localized fucosyltransferase later named FUCOSYLTRANSFERASE 1 (FUT1) (ref. 6). In contrast to mur1 mutants, no defects in growth or cell wall strength were observed in mur2 plants. Similarly, fut4 and fut6 mutants that are defective in arabinogalactan fucosylation do not exhibit phenotypic differences under standard growth conditions when compared with wild-type plants 8,16 .

All GDP sugars are biosynthesized in the cytosol and require transport into the Golgi or ER lumen to be made available for glycosylation reactions. To date, a number of nucleotide sugar transporters (NSTs) have been characterized in plants ${ }^{17-22}$. However, the only GDP sugar transporter unambiguously characterized is the GDP-Man transporter GOLGI-LOCALIZED NUCLEOTIDE SUGAR TRANSPORTER 1 (GONST1) from Arabidopsis. GONST1 was initially identified based on its homology to GDP sugar transporters identified in other organisms $s^{23}$. Several studies have established that GONST1 localizes to the Golgi apparatus, and that it can functionally complement the yeast vanadate resistance glycosylation ( $v r g 4-2)$ GDP-Man transporter mutant ${ }^{23,24}$. More recently, it has been shown that GONST1 can transport multiple GDP sugars in vitro, and that gonst 1 mutants are dwarfed and can develop spontaneous leaf lesions; however, their cell walls are undistinguishable from wild-type plants ${ }^{2}$. A more detailed characterization of the gonst 1 mutant revealed that GIPC head groups are altered in the mutant, indicating that GONST1 function is essential for the mannosylation of GIPCs in vivo ${ }^{2}$. The loss-of-function mutant analyses also suggests that GONST1 is a specific GDP-Man transporter, which is most likely not responsible for the transport of GDP-Fuc and other GDP sugars in planta.

The phenotypes obtained for the murl mutants indicate that the availability of GDP-Fuc is critical for normal plant development. Since GDP-Fuc is synthesized in the cytosol but is utilized in the Golgi lumen, a transporter is required to cross the Golgi membrane. To date, very little is known regarding the transport of this GDP sugar in plants other than a requirement for GDP-Fuc transport for the fucosylation of xyloglucan ${ }^{25}$. This study identifies and biochemically characterizes an Arabidopsis Golgi-localized GDP-Fuc transporter (AT5G19980), previously named GONST4 according to its homology to other GDP sugar transporters. We show that GONST4 specifically transports GDP-Fuc and not GDP-Man, and our in vivo analysis supports its role as the primary Golgi GDP-Fuc transporter. Due to this newly attributed function, we have revised its name to GDP-fucose transporter 1 (GFT1).

\section{Results}

GFT1 and the Arabidopsis NST family. The previously identified GONST sub-family is part of clade III of the NST/TPT family ${ }^{20}$. A phylogenetic analysis of the full-length protein sequences of the Arabidopsis NST clade III members and the previously characterized Saccharomyces cerevisiae (yeast) GDP-Man transporter VRG4 (ref. 26) and its paralogue, which is termed homologous to VRG4 (HVG1), indicate that both GONST1 (AT2G13650) and GONST2 (AT1G07290) cluster together with VRG4 and HVG1 to form a sub-clade (A), while GONST3 (AT1G76340) and GFT1 (AT5G19980) form a sub-clade (B). A further sub-clade (C) contains two additional candidates, one of which UTr7 (AT4G31600) has been identified as a UDP-Glc/ UDP-Gal transporter ${ }^{27}$ (Fig. 1). GFT1 shares $49 \%$ identity with GONST3, and 22 and $21 \%$ identity to GONST1 and GONST2,

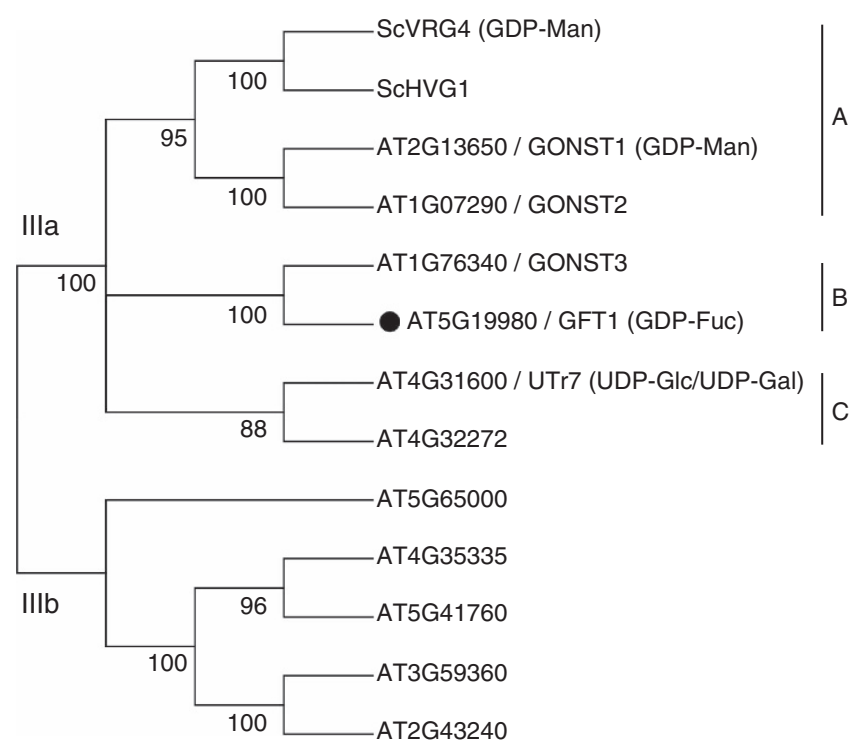

Figure 1 | Phylogenetic tree of the Arabidopsis NST sub-family III.

Full-length amino-acid sequences were aligned and the phylogenetic tree generated using MEGA6. Clades were assigned as previously reported 20 . The yeast GDP-Man transporter ScVRG4 and its paralogue ScHVG1 have also been included in the phylogenetic analysis. The sub-clade Illa contains the previously characterized Arabidopsis GDP-Man transporter GONST1 (ref. 23) and UDP-Glc/UDP-Gal transporter UTr7 (ref. 27). Only bootstrap values $>50 \%$ are shown. 
respectively. In comparison, GONST1 and GONST2 share $64 \%$ identity at the amino-acid level, while across species, GONST1 shares 31\% identity with VRG4 and 32\% identity with HVG1. Members of sub-clade (C) share $9-21 \%$ identity in their amino-acid sequences when compared with all other members of clade III of the Arabidopsis NST family.

GFT1 is ubiquitously expressed and localizes to the Golgi. In silico expression analysis of publicly available microarray data comprising the AtGenExpress developmental data set ${ }^{28}$ revealed relatively high and ubiquitous expression of GFT1. Similarly, GONST1 and GONST3 are ubiquitously expressed at slightly lower levels compared with GFT1. In contrast, the GONST2 transcript appears to be expressed at very low levels with slightly elevated expression in mature pollen (Fig. 2a). These expression levels are also reflected in the total expressed sequence tag (EST) counts for the GONST clade members. GFT1, GONST1 and GONST3 are all represented by $>20$ ESTs, while there is only a single EST allocated to the GONST2 locus. To establish and confirm the sub-cellular localizations of GFT1 and remaining GONST clade members, we generated C-terminal yellow fluorescent protein (YFP) fusions and expressed them transiently in Nicotiana benthamiana leaves. The GFT1, GONST1 and GONST2 fusion proteins localized to Golgi-like punctate structures and co-localized with the Golgi marker $\alpha$ mannosidase I (aMan; Fig. 2b). These results confirm previous Golgi localizations for GONST1 (ref. 23) and GFT1 (refs 24,29). Despite several attempts, we were unable to express and localize GONST3-YFP in N. benthamiana leaves using this transient localization assay.

Establishing the in vitro function for GFT1. To assess GFT1 function, we heterologously expressed the protein in yeast and determined its capacity to transport nucleotide sugars using a recently established approach ${ }^{20,2}$. Previous work had indicated that GFT1 had the capacity to partially complement the yeast GDP-Man transporter mutant vrg4 (ref 24). As a consequence, we also assessed the ability of the previously characterized GDP-Man transporter, GONST1 and its paralogue GONST2 to transport nucleotide sugars using the in vitro transporter assay. Unfortunately, as previously outlined for the transient localization assay, we were also unable to express the GONST3 protein in yeast. The corresponding genes were expressed in yeast and microsomal proteins were reconstituted into liposomes for transporter assays. Immunoblot analysis confirmed the presence of each protein (Fig. 3a). Subsequently, proteo-liposomes pre-loaded with either uridine- $5^{\prime}$-monophosphate (UMP), guanosine- $5^{\prime}$-monophosphate (GMP), cytidine- $5^{\prime}$-monophosphate (CMP) or adenosine- $5^{\prime}$-monophosphate (AMP) were incubated with a mixture of 16 nucleotide/nucleotide sugar substrates (Fig. 3b). Non-incorporated substrates were removed by gel filtration, and the content of the liposomes after in vitro transport was analysed by liquid chromatography-tandem mass spectrometry (LC-MS/MS; Fig. 3c, d). Our results confirmed the previous finding that GONST1 functions preferentially as a GDP-Man transporter ${ }^{23}$ and perhaps as a general GDP sugar transporter with the capacity to transport comparably lower amounts of GDP-Fuc, GDP-Glc and GDP-Gal (Fig. 3e), as has been recently reported ${ }^{2}$. In contrast, GONST2 was limited in its capacity to transport any NDP sugar substrates using the assay (Fig. 3e). Although transport of GDP sugars could be observed in the GONST2 assay, this is likely the result of endogenous transporter activity present in the yeast microsomal preparations, since the incorporation levels were similar to those observed in control reactions (yeast transformed with the empty vector). Our analysis of GFT1 indicated that this NST exhibited the highest activity with GDP-Fuc in comparison with the control, GONST1 and GONST2. The data also show that GFT1 preferentially transported GDP-Fuc in our in vitro assay, since no side activities with GDP-Man or GDP-Glc were observed. A minor activity was observed with GDP-Gal, but this was quite low in comparison with the transport of GDP-Fuc (Fig. 3e). All transport was strictly dependent on the presence of GMP as exchange substrate, since no significant transport was observed when proteo-liposomes were pre-loaded with UMP, CMP or AMP (Supplementary Fig. 1). Furthermore, only GMP was accepted as exchange substrate when proteo-liposomes were pre-loaded with GDP-Fuc and then incubated with a mix of the four NMPs (Fig. 3f). No significant transport of any NMP was observed in GFT1containing proteo-liposomes pre-loaded with GDP-Man (Fig. 3g).

Kinetic parameters of GFT1 and GONST1. To support an in vivo role for GFT1 as the Arabidopsis GDP-Fuc transporter, we undertook a detailed examination of the kinetics of GDP-Fuc transport. The GFT1-mediated transport of GDP-Fuc was saturable in a concentration- and time-dependent manner (Fig. 4a,b). The determination of kinetic parameters for GFT1 revealed an apparent $K_{\mathrm{m}}$ for GDP-Fuc of $7 \mu \mathrm{M}$ with a turnover rate of $\sim 4 \mathrm{~s}^{-1}$ (Table 1). The apparent $K_{\mathrm{m}}$ of GONST1 for GDP-Fuc was 76 and $17 \mu \mathrm{M}$ for GDP-Man (Table 1).
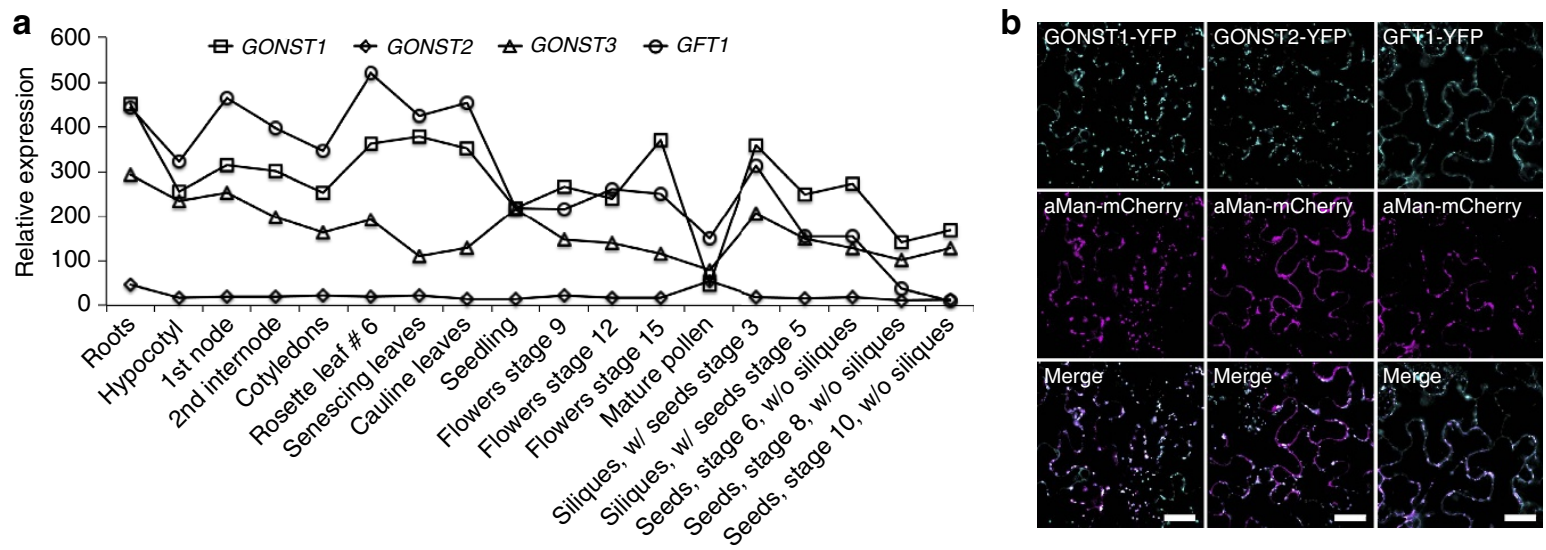

Figure 2 | Expression pattern and sub-cellular localizations of Arabidopsis GONST members. (a) Expression patterns of GFT1 and GONST1-3 in different Arabidopsis organs and developmental stages using the AtGenExpress developmental data set ${ }^{28}$. (b) Sub-cellular localizations of GFT1, GONST1 and GONST2. C-terminal YFP fusion constructs were transiently co-expressed with the aMan-mCherry Golgi marker in N. benthamiana leaves. Scale bar, $25 \mu \mathrm{m}$. 

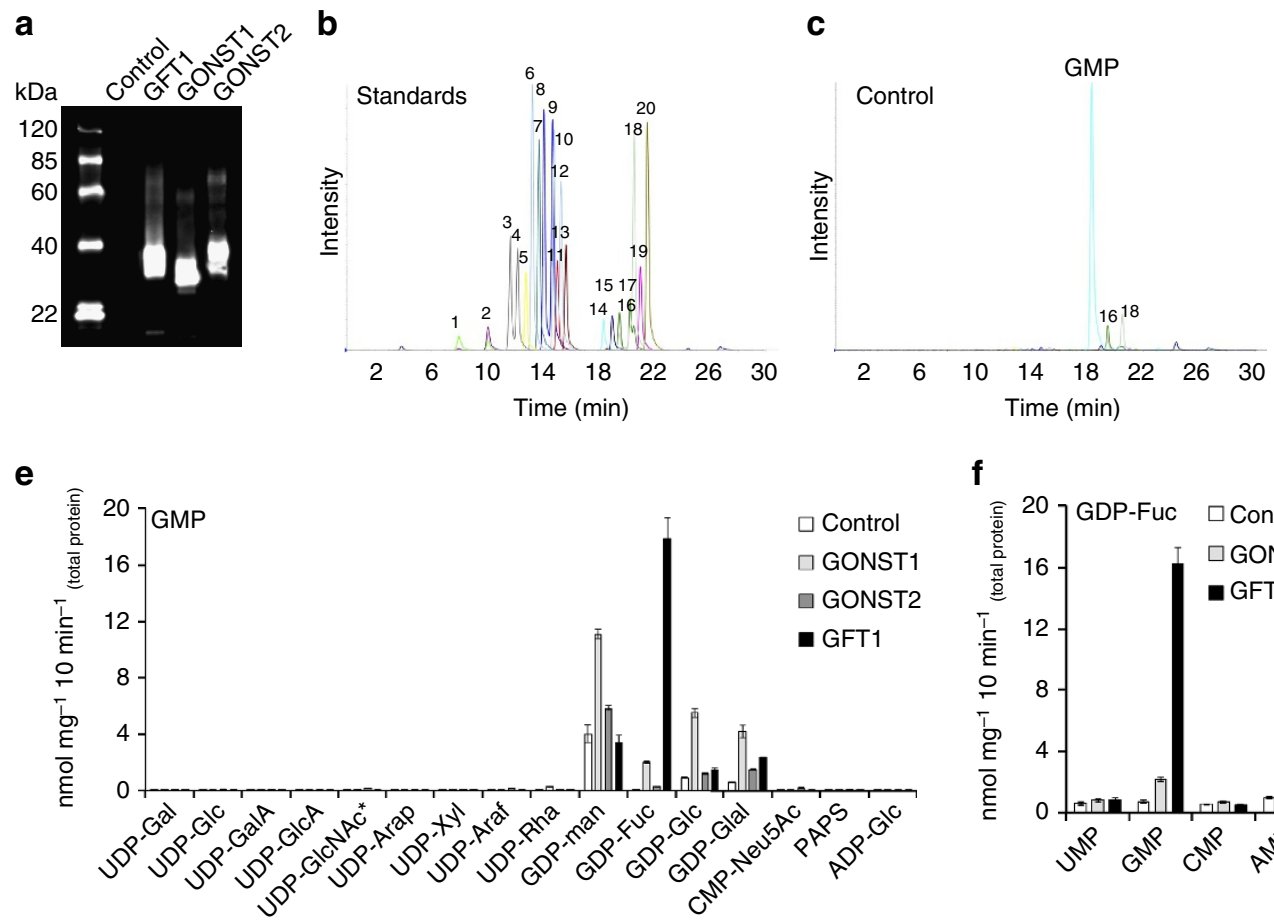

d

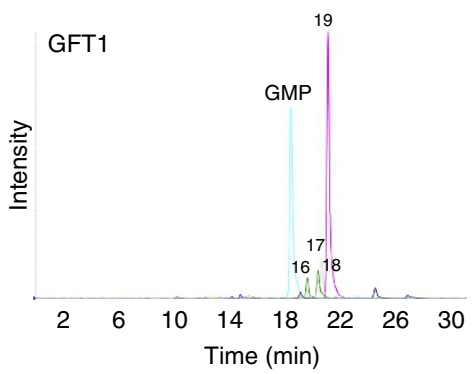

f

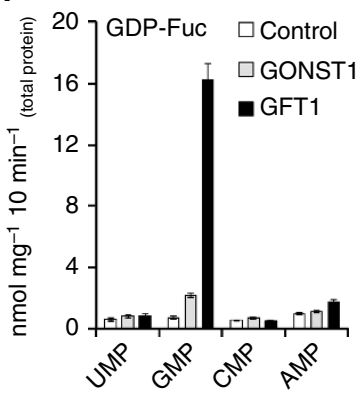

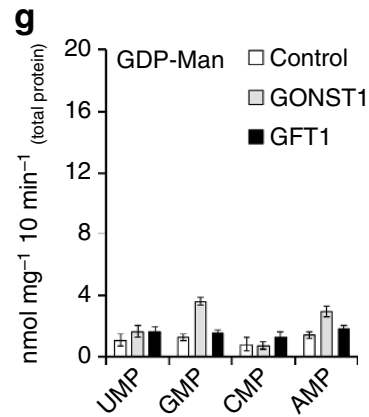

Figure 3 | LC-MS/MS analysis of nucleotide sugar transport activities for GFT1 and other GONST members. (a) Immunoblot analysis of empty vector, GFT1, GONST1 and GONST2 expression in proteo-liposomes (2.5 $\mu$ g protein per lane). (b) Separation of a 20 nucleotide/nucleotide sugar mix (1, CMP; 2 , UMP; 3, UDP-GalA; 4, UDP-GlcA; 5, CMP-Sia; 6, UDP-Arap; 7, UDP-Rha; 8, UDP-Gal; 9, UDP-Glc; 10, UDP-Xyl; 11, UDP-GlcNAc/GalNAc; 12, UDP-Araf; 13, PAPS; 14, GMP; 15, AMP; 16, GDP-Man; 17, GDP-Gal; 18, GDP-Glc, 19, GDP-Fuc; 20, ADP-Glc). LC-MS/MS analysis of proteo-liposomes derived from (c) yeast transformed with the empty vector (control) or (d) yeast expressing GFT1 after simultaneous incubation with 16 nucleotide sugar substrates. (e) Quantification of nucleotide sugar transport into proteo-liposomes pre-loaded with $10 \mathrm{mM} \mathrm{GMP}$. (f and $\mathbf{g}$ ) Exchange substrate specificities of GFT1 and GONST1. Proteo-liposomes were loaded with $1 \mathrm{mM}$ GDP-Fuc (f) or $1 \mathrm{mM}$ GDP-Man (g) and incubated with a mix of UMP, GMP, CMP, AMP at a concentration of $200 \mu \mathrm{M}$. Data are mean and s.d. of $n=4$ assays.
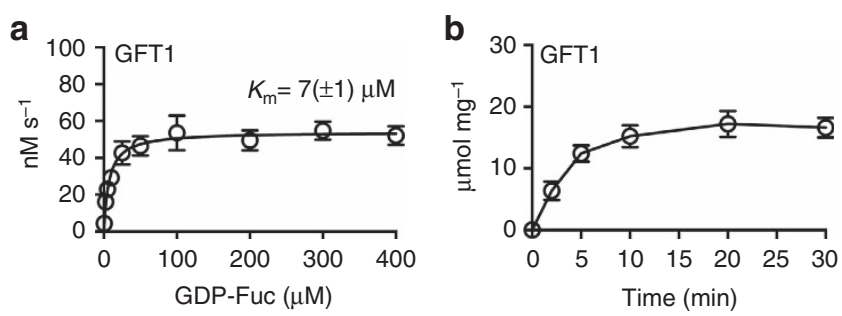

Figure 4 | Concentration- and time-dependent transport activities for GFT1. (a) Proteo-liposomes, pre-loaded with $10 \mathrm{mM} \mathrm{GMP}$, were incubated with GDP-Fuc at varying concentration $(0.5-400 \mu \mathrm{M})$ for 2 min at $25^{\circ} \mathrm{C}$. (b) Proteo-liposomes, pre-loaded with $10 \mathrm{mM} \mathrm{GMP}$, were incubated with GDP-Fuc at a concentration of $50 \mu \mathrm{M}$ for the indicated time points at $25^{\circ} \mathrm{C}$. Values are normalized to the actual NST content present in proteo-liposome preparations (Supplementary Table 1). Data are the mean and s.e.m. of $n=4$ assays.

This reported $K_{\mathrm{m}}$ for GDP-Man is consistent with a previous report that estimated $K_{\mathrm{m}}$ of $26 \mu \mathrm{M}$ for GONST1 for GDP-Man ${ }^{30}$. Previously, we determined the GDP-Fuc content in various Arabidopsis organs ${ }^{20}$. GDP-Fuc concentrations are in the range of 5-20 pmol mg ${ }^{-1}$ dry weight, while the GDP-Man concentrations range from 10 to $50 \mathrm{pmol} \mathrm{mg}^{-1}$ dry weights. Assuming the plant vacuole comprises the majority of the aqueous content of a plant cell, the dry weight would be equivalent to the volume of the cellular contents $(\mu \mathrm{l})$. Accordingly, these cellular GDP-Fuc and GDP-Man concentrations can be considered to be approximately in the $\mu \mathrm{M}$ range. Thus, we estimate that the $K_{\mathrm{m}}$ value for GDP-Fuc of GFT1 $(7 \mu \mathrm{M})$ determined using the in vitro assay is

\section{Table 1 | Kinetic parameters of GDP-Fuc and GDP-Man} transport into proteo-liposomes.

\begin{tabular}{lcccc}
\multirow{2}{*}{ Parameter } & \multicolumn{2}{c}{ GDP-Fuc } & & GDP-Man \\
\cline { 2 - 3 } \cline { 5 - 5 } & GFT1 & GONST1 & & GONST1 \\
\hline$K_{\mathrm{m}}(\mu \mathrm{M})$ & $7(1)$ & $76(5)$ & & $17(3)$ \\
$V_{\max }\left[\mathrm{nMs} s^{-1}\right]$ & $54(1)$ & $14(0)$ & & $24(1)$ \\
$k_{\text {cat }}\left(\mathrm{s}^{-1}\right)$ & 4.0 & 0.7 & & 1.2
\end{tabular}

For each transporter (GFT1 or GONST1), data points with varying substrate concentrations $(0.5-400 \mu \mathrm{M})$ were acquired. Values are mean (s.e.m.) of $n=4$.

within physiological range. Similarly, the $K_{\mathrm{m}}$ value for GDP-Man of GONST1 $(17 \mu \mathrm{M})$ would also be consistent with physiological concentrations. However, the $K_{\mathrm{m}}$ value for GDP-Fuc transport of GONST1 $(76 \mu \mathrm{M})$ is significantly higher than the physiological concentration of GDP-Fuc, indicating that GONST1 does not likely play a significant role as GDP-Fuc transporter in vivo.

The role of GFT1 in planta. To evaluate the in vivo function of GFT1, two putative T-DNA insertion lines were obtained with predicted insertions in the single exon at locus AT5G19980. However, we were unable to identify a T-DNA insertion within the corresponding SAIL_400_E04 and SALK_094857 populations. As a consequence, we used an RNA interference approach through the construction of a hairpin construct specifically targeting GFT1 transcripts. A total of 75 independent hairpin GFT1 lines (hpGFT1) were selected, and classified into four categories (cohorts) each of which were characterized by 
a

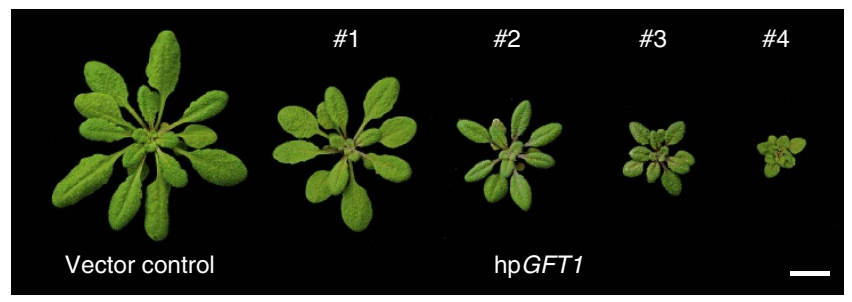

b

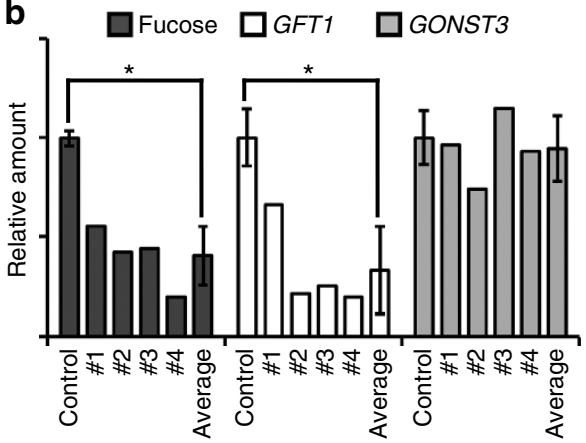

Figure 5 | Morphological phenotypes and biochemical characterization of hpGFT1 lines. (a) Representative phenotypes (cohorts) of plants transformed with a hairpin construct specifically targeting GFT1 expression compared with control plants (plants transformed with the empty vector). Resultant plants were assigned to four categories based on the severity of the phenotype. Scale bar, $10 \mathrm{~mm}$ (b) Total L-fucose content from cell wall extracts (Fucose) derived from hpGFT1 lines and the corresponding reductions in GFT1 expression in these lines. GONST3 expression was not significantly affected in the hpGFT1 lines. The control values are mean and s.d. of three independent measurements $(n=3)$. The values for the hpGFT1 lines are the mean of each cohort analysed in triplicate. The 'average' is the mean and s.d. $(n=4)$ of the data from the four independent hpGFT1 cohorts. Fucose content $(P<0.003)$ and GFT1 transcript levels $(P<0.005)$ are significantly different by Student's $t$-test. Values are expressed relative to control lines (plants transformed with the empty vector).

varying growth phenotypes (Fig. 5a). Remarkably, $\sim 50 \%$ of the plants died after selection. These plants displayed the strongest morphological defects (10 days post germination) and exhibited severe growth inhibition before death. Analysis of GFT1 transcript levels from lines in these four categories using quantitative PCR revealed significant reductions in GFT1 expression correlating with the observed severe developmental defects compared with the control plants transformed with the empty vector (Fig. 5). In comparison, transcript levels of GONST3, the closest GFT1 homologue, were not significantly affected in these plant lines indicating that the hpGFT1 construct specifically targets GFT1 expression (Fig. 5b). The reductions in the amounts of GFT1 transcripts and presumably GDP-Fuc transport did not affect the nucleotide sugar levels, specifically the levels of GDPFuc, in hpGFT1 plants (Supplementary Table 2).

Analysis of the monosaccharide composition of cell wall extracts revealed considerable reduction in total cell wall-derived L-fucose content (up to $80 \%$ ) that correlated with the observed reduction in GFT1 expression (Fig. 5b). To investigate which cell wall polymers are most affected by the downregulation of GFT1 expression, we determined the monosaccharide composition of sequentially extracted cell wall material derived from hpGFT1 lines (Supplementary Table 3). The analysis revealed a significant reduction $(\sim 70 \%)$ in the $\mathrm{L}$-fucose content in the pectin-enriched CDTA and $\mathrm{Na}_{2} \mathrm{CO}_{3}$ fractions. The reduction in cell wall $\mathrm{L}$-fucose levels was further detected in the xyloglucan-enriched fractions ( 1 and $4 \mathrm{~N} \mathrm{KOH}$ ). Moreover, in the $4 \mathrm{~N} \mathrm{KOH}$ fractions of the most severe line hpGFT1\#4, no residual L-fucose could be detected (Supplementary Table 3). While a significant increase in the proportion of galactose (Gal) in the $4 \mathrm{~N} \mathrm{KOH}$ fractions was observed in the hPGFT1 lines, no other sugar varied. Results from the compositional analysis of fractionated cell wall material were further confirmed by oligosaccharide mass profiling (OLIMP). To understand how the reduction in L-fucose content specifically affects the composition of xyloglucan, we enzymatically digested cell wall material from the hpGFT1 lines with a xyloglucanase and analysed the solubilized oligosaccharide mixtures using matrix-assisted laser desorption/ionization time-of-flight mass spectrometry. The results show that the xyloglucan profiles from hpGFT1 lines clearly differ from those of control plants transformed with the empty vector. The relative abundance of fucosylated oligosaccharides such as XXFG and XLFG as well as the corresponding acetylated versions XXFG and $\mathrm{XLFG}^{31}$ released from cell wall material of the hpGFT1 lines was significantly decreased compared with the control. Notably, in the lines with the strongest reduction of GFT1 expression (hpGFT1\#4), no fucosylated oligosaccharides were detectable (Fig. 6; Supplementary Table 4). Concurrent with the reduced abundance of fucosylated oligosaccharides, non-fucosylated but galactosylated oligosaccharides (XXLG/XLXG and XLLG/XXJG) increased in the lines with reduced GFT1 expression. The OLIMP analysis was unable to determine whether L-galactose was present in xyloglucan in the hpGFT1 lines due to the similarities in oligosaccharide masses. The resultant L-galactose containing oligosaccharide (XXJG) has previously been shown to occur in mur1 mutants ${ }^{13}$.

To examine whether GFT1 plays a role in the fucosylation of proteins, we analysed the monosaccharide content of total protein extracted from hpGFT1 lines from phenotypic categories 1,2 and 3 (Fig. 7). A clear reduction in L-fucose was observed in the protein extracts indicating reduced protein fucosylation. Cell wall contamination from pectic material was minimal based on the absence of rhamnose in these samples. Last, to specifically determine the impact on fucosylation of $N$-linked glycans, we analysed total protein with antibodies raised against plant $\mathrm{N}$-glycan xylosyl and fucosyl epitopes (Supplementary Fig. 2). As anticipated, xylosylation levels of glycoproteins in the hpGFT1 lines were not correlated with the severity of the phenotypes. Overall, when compared with control plants transformed with the empty vector, we observed reductions in the fucosylation signal from proteins derived from hpGFT1 lines from the three categories concomitant with the severity of the observed phenotype (Fig. 5a). The results indicate that the reduction in GFT1 expression also had a clear impact on the levels of protein fucosylation in plants.

\section{Discussion}

A family of NST-related proteins from Arabidopsis termed GONST2, 3, 4 (GFT1) and 5 had been previously been assigned as putative GDP-Man transporters based on the sequence identity to the GDP-Man transporter GONST1 (ref. 24). Previous work had identified GONST1 and GONST2 as GDP-Man transporters and speculated that GFT1 (GONST4) could transport GDP-Man. However, since it could not completely rescue the GDP-Man transport defective yeast mutant $v$ rg4-2, it was concluded that it 


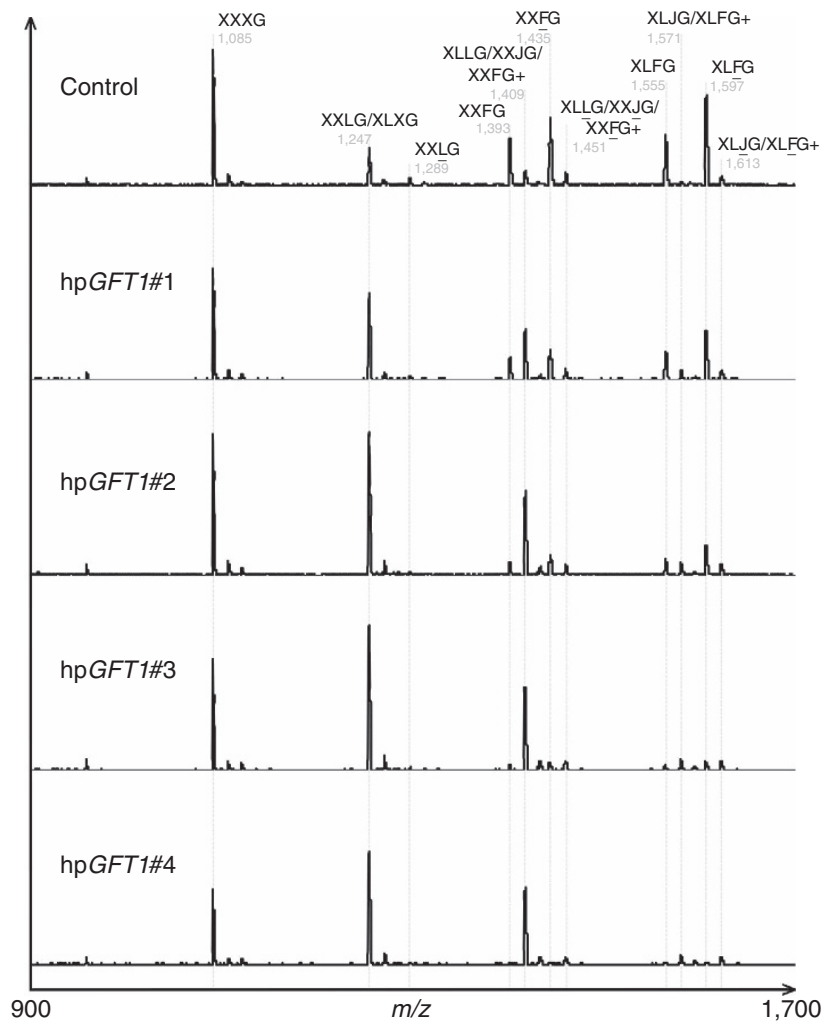

Figure 6 | Xyloglucan structures in hpGFT1 lines. Abundances of xyloglucan oligosaccharides in cell wall material derived from hpGFT1 lines were determined by oligosaccharide mass profiling (OLIMP). One-letter code nomenclature of oligosaccharides according to ref. 32. All masses are $\left[\mathrm{M}+\mathrm{Na}^{+}\right]$except those labelled $(+)$which indicates $\left[\mathrm{M}+\mathrm{K}^{+}\right]$. The analysis was carried out in triplicate (Supplementary Table 4).

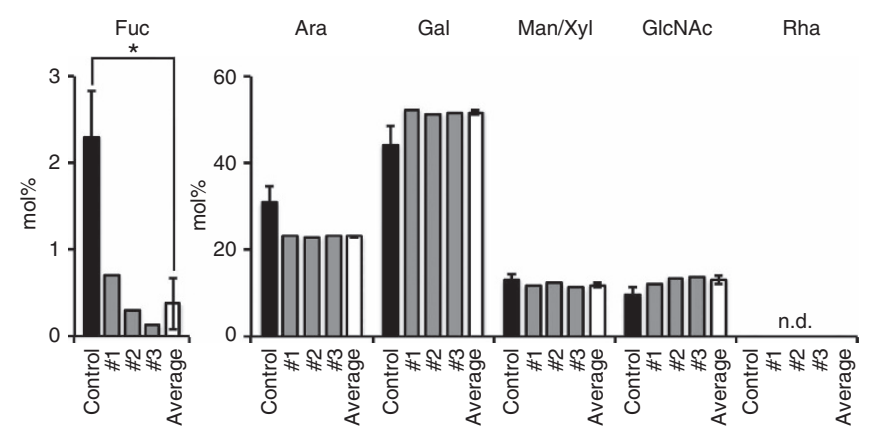

Figure 7 | Protein fucosylation in hpGFT1 lines. Monosaccharide content (mol \%) of trifluoroacetic acid-hydrolysed total protein extracted from hpGFT1 lines measured by anion exchange chromatography. The control values are mean and s.d. of four independent analyses $(n=4)$. The values for the hpGFT1 lines represent the mean of each cohort analysed in triplicate. The 'average' is the mean and s.d. $(n=3)$ of data from the three independent hpGFT1 cohorts. Fuc levels are significantly different $(P<0.0004)$ by Student's $t$-test, as were the sugars Ara $(P<0.009)$, Gal $(P<0.02)$, Man $/ \mathrm{Xyl}(P<0.03)$ and GlcNAc $(P<0.005)$ to a lesser degree.

may transport other nucleotide sugars in planta ${ }^{24}$. In this study, we have shown that GFT1 (GONST4) is a Golgi-localized transporter with preferential transport activity for GDP-Fuc in plants. We also present evidence that GFT1 function is indispensable for plant growth and development, since plants with significantly reduced GFT1 expression are severely dwarfed and do not grow normally.
The sub-cellular localization of GFT1, GONST1 and GONST2 indicated that all three proteins are located in the Golgi apparatus. This localization is consistent with their function as Golgi NSTs, since the synthesis of D-mannose and L-fucose-containing glycans occurs in this compartment ${ }^{6,32}$. Our localization data also confirm previous findings for GFT1 and GONST1 (ref. 24); however, this represents the first localization evidence for GONST2. Given the assigned functional role for GFT1 in transporting GDP-Fuc for lumenal fucosylation reactions, we wanted to confirm its Golgi location and exclude compartments, such as the ER, that could potentially also support this function. Consequently, we re-assessed the sub-cellular localization of GFT1 in $N$. benthamiana using both ER and Golgi markers (Supplementary Fig. 3). We observed a consistent overlap of GFT1-YFP with the Golgi marker with scatter plots indicating a positive correlation. This was further supported by a thresholded Manders' coefficient (tM1 and tM2) analysis, which indicated a high level of overlap between fluorescent signals (Supplementary Fig. 3c). In contrast, we observed a poor correlation between GFT1-YFP and the ER marker (Supplementary Fig. 3b), which was further confirmed after a thresholded Manders' coefficient analysis (Supplementary Fig. 3c).

The transcripts for GONST1, GONST3 and GFT1 are found ubiquitously throughout plant development (Fig. 2). In contrast, GONST2 expression levels appear to be very low, which is also supported by the scarce EST evidence, suggesting that GONST2 may not have a major role in most plant organs with the exception of those where some expression is observed, such as mature pollen. Indeed, while GONST2 appeared to be capable of partially complementing the yeast GDP-Man transporter mutant vrg4-2 (ref. 24), the in vitro assay demonstrated negligible GDP-Man transport and our results show that it is incapable of in vitro transport of GDP-Fuc. While GONST2 exhibits minimal expression, GONST3 is expressed throughout the plant and is homologous to GFT1 with a $49 \%$ identity. The gene model, which contains no intron, is supported by multiple ESTs and a complementary (cDNA), but despite repeated attempts, we were unable to clone GONST3 from cDNA according to this gene model. Similar difficulties have been reported previously ${ }^{24}$, which is consistent with our results and suggests that the annotated gene model for GONST3 may be incorrect. Interestingly, a recent report biochemically characterized the GONST3 orthologue from grapevine (VvGONST-A) as a GDP-Man transporter ${ }^{33}$, perhaps providing some functional evidence for this locus in Arabidopsis. On the other hand, the same study also reports that a grapevine orthologue of GFT1 (VvGONST-B), is a GDP-Glc transporter and exhibits no GDP-Fuc transport ${ }^{33}$. Since the two proteins share high levels of sequence identity (71\%), it would be interesting to assess VvGONST-B using our transport assay. It appears that GFT1-like proteins are ubiquitously present in plants; however, mammalian genomes do not appear to contain orthologues of GFT1. Instead, the protein SLC35C1 has been shown to be a GDP-Fuc transporter in mammalian cells ${ }^{34}$ and this protein does not have any close homologues in plants. Thus, the specificity for GDP-Fuc transport has apparently evolved convergently in animals and plants.

To support the in vitro function of GFT1, we attempted to identify loss-of-function mutants. However, we were unable to confirm a corresponding T-DNA insert in either of the available lines, both of which had predicted inserts in the single exon of the GFT1 locus. To overcome this lack of an appropriate knockout line, we generated an RNA interference construct specifically targeting GFT1 transcripts. Analysis of randomly selected plant lines by quantitative PCR from four phenotypic categories 
revealed varying degrees of suppression of GFT1 expression. Consistent with the specific in vitro GDP-Fuc transport activity, the reduction in GFT1 expression strongly correlated with a reduction in L-fucose in both cell wall components as well as glycoproteins in hpGFT1 lines. The observed relationship between reduced GFT1 expression, the measured impact on fucosylation in the plant, and the resultant severe developmental defects indicate that GFT1 is likely the sole GDP-Fuc transporter in Arabidopsis. Collectively, these data support the notion that GFT1 cannot be substituted by GONST1, GONST2 or GONST3 in planta.

Most of the L-fucose in plant cell walls is present in pectin and xyloglucan. The $\mathrm{L}$-fucose content in cell wall extracts of hpGFT1 lines revealed significant reductions in pectin- and xyloglucanenriched fractions, while a more detailed analysis of xyloglucan structures indicated reduced levels of L-fucose-containing oligosaccharides. The reduction in cell wall $\mathrm{L}$-fucose, in combination with the developmental abnormalities observed in plant lines with the strongest reduction in GFT1 expression are reminiscent of the mur1 mutant ${ }^{12,35,36}$. mur1 plants almost completely lack L-fucose due to a loss-of-function mutation in the GMD2 gene, which is required for GDP-Fuc biosynthesis. Similar to our results, mur1 plants contain significantly reduced levels of L-fucose in pectic fractions $s^{35}$ and have decreased $\mathrm{L}$-fucose-containing oligosaccharides and a concomitant increase in non-fucosylated Gal-containing oligosaccharides derived from xyloglucan ${ }^{12}$. It has been previously shown that the murl plant developmental phenotype is specifically associated with an altered structure of RG-II, since other mutations that solely affect fucosylation of xyloglucan or arabinogalactan proteins do not show growth defects under standard growth conditions $s^{6,8,16}$. The hpGFT1 lines also had a decrease in the amount of L-fucose in $\mathrm{N}$-linked glycans. The analysis of $\mathrm{N}$-glycans from the murl mutant showed that almost $95 \%$ of L-fucose residues were absent ${ }^{14}$. Mutants affected in fucosylation of $\mathrm{N}$-glycans such as the hybrid glycan $1(\mathrm{hgll})$ or complex glycan 1 (cgl1) revealed no major physiological phenotype $^{37}$ besides reduced root growth under salt stress ${ }^{38}$. These observations further support the importance of L-fucose for the correct organization of pectic polysaccharides and their role in plant growth and development. Thus, the severe morphological defects of the hpGFT1 lines are likely explained by the reduced fucosylation of the pectic polymer RG-II.

The similarity in phenotype between the hpGFT1 knockdown lines and the mur 1 knockout mutant is likely due to the existence of a second GMD isoform (GMD1) in Arabidopsis. While GMD2 is ubiquitously expressed in the aboveground portions of the plant, GMD1 is only expressed in stipules and pollen grains ${ }^{39}$. Therefore, mur 1 mutants are likely to contain residual GDP-Fuc, which enables the plants to survive. This residual functionality in the hpGFT1 lines also explains the similarities to the murl phenotype. However, it is very likely that the complete lack of GFT1 expression or function would lead to a complete loss of glycan fucosylation and a lethal phenotype. This is in agreement with our observation that $>50 \%$ of the $h p G F T 1$ population (total of 75 independent plant lines) died at early developmental stages. These results strongly suggest that GFT1 may be the only GDP-Fuc transporter in Arabidopsis.

It was generally assumed that the structural alterations to RG-II in the murl mutant were mainly due to the substitution of L-fucose by L-galactose ${ }^{35}$; however, it now appears that the altered phenotype is a result of a truncation of chain A from RG-II due to the lack of available GDP-Fuc ${ }^{9}$. It is thus highly likely that the hpGFT1 lines possess a similar and truncated form of RG-II. Since we observed a significant increase in Ara content, it is possible that the hpGFT1 plants were compensating RG-II truncations by increasing arabinan side chains on RG-I.
Overall, the similarities between the hpGFT1 lines and the murl mutant support the exclusive role of GFT1 in the transport of GDP-Fuc in Arabidopsis. Thus, the impairment of cytosolic-derived GDP-Fuc biosynthesis in plants is akin to the inhibition of GDP-Fuc transport into the Golgi apparatus.

We have identified and characterized a plant Golgi-localized nucleotide sugar transporter that transports GDP-Fuc in vitro. This is the first instance where a NST with specificity for GDP-Fuc has been identified in plants. hpGFT1 mutant plants showed a $\sim 80 \%$ decrease in total cell wall $\mathrm{L}$-fucose content affecting pectins as well as xyloglucans, thus confirming the role of GFT1 in providing GDP-Fuc for cell wall biosynthesis. Fucosylation of glycoproteins was also impaired in hpGFT1 mutants. Plants with significantly diminished GFT1 expression were severely dwarfed and did not grow normally, which indicates that GFT1 plays an essential role in plant growth and development.

\section{Methods}

Substrates. Substrates were obtained from the following sources: UDP- $\alpha$-D-xylose, UDP- $\beta$-L-arabinopyranose and UDP- $\alpha$-D-galacturonic acid (Carbosource Services, Complex Carbohydrate Research Center, Athens, GA); UDP- $\alpha$-D-glucuronic acid, UDP- $\alpha$-D-glucose, UDP- $\alpha$-D-galactose, UDP- $N$-acetyl$\alpha$-D-glucosamine, UDP- $N$-acetyl- $\alpha$-D-galactosamine, GDP- $\alpha$-D-mannose, GDP- $\beta$-L-fucose, GDP- $\alpha$-D-glucose, adenosine $3^{\prime}$-phosphate $5^{\prime}$ phosphosulfate, CMP- $N$-acetylneuraminic acid and ADP- $\alpha$-D-glucose (Sigma-Aldrich, St Louis, $\mathrm{MO}$ ); and UDP- $\beta$-L-arabinofuranose (Peptides International, Louisville, KY). GDP- $\alpha$-L-Galactose was synthesized enzymatically using the Arabidopsis GDP-mannose-3,5-epimerase (GME, At5g28840) essentially as outlined ${ }^{40}$. The GME cDNA was cloned into pET-DEST42 and expressed in Escherichia coli BL21 Star (DE3) strain (Thermo Fisher Scientific). The His-tagged GME was then purified from lysates using TALON Metal Affinity Resin (Clontech) and desalted using PD-10 Desalting Columns (GE Healthcare). GDP-Gal was synthesized using $100 \mu \mathrm{g}$ of purified GME, $10 \mathrm{mM}$ GDP-Man and $50 \mathrm{mM}$ Tris ( $\mathrm{pH} \mathrm{8.0)}$, and incubated overnight at $25^{\circ} \mathrm{C}$. The resultant GDP-Gal was purified from the remaining GDP-Man and GDP-L-gulose (also biosynthesized by GME) using high-performance liquid chromatography (HPLC) performed using a Dionex Ultimate 3000 with ultraviolet light detection at $262 \mathrm{~nm}$. The reaction was separated on a CarboPac PA20 column $(3 \times 150 \mathrm{~mm}$; Dionex $)$ at a flow of $0.5 \mathrm{ml} \mathrm{min}^{-1}$ with a linear gradient of $50 \mathrm{mM}$ to $1 \mathrm{M}$ ammonium formate over $40 \mathrm{~min}$ (ref. 41). Peaks corresponding to GDP-Gal were concentrated using a vacuum concentrator. UDP- $\beta$-L-rhamnose was enzymatically synthesized by a two-step reaction from UDP-Glc using the 4,6-dehydratase (RHM-1D) and the 3,5-epimerase 4-reductase (RHM1-ER) domains of Arabidopsis UDP-rhamnose synthase (RHM1, Atlg78570). The domains were cloned into pET11a (Novagen) and expressed in E. coli Rosetta 2 (DE3) strain (EMD Millipore), and purified from lysates using TALON Metal Affinity Resin (Clontech). The initial reaction to produce UDP-D-quinov-4-ulose was synthesized using $1 \mathrm{mM}$ UDP-Glc, $4 \mu \mathrm{g}$ RHM-1D, $0.5 \mathrm{mM} \mathrm{NAD}^{+}$and $10 \mathrm{mM}$ Tris-HCL (pH 8.5) in a total volume of $0.4 \mathrm{ml}$, and incubated overnight at $25^{\circ} \mathrm{C}$. The volume of the reaction mixture was adjusted to $2 \mathrm{ml}$, using a solution containing $1 \mathrm{mM} \mathrm{NADPH}, 8 \mu \mathrm{g}$ RHM1-ER and $10 \mathrm{mM}$ Tris- $\mathrm{HCl}(\mathrm{pH} 8.5)$, and incubated overnight at $25^{\circ} \mathrm{C}$. The resultant UDP-Rha was purified using an ENVI-Carb SPE column (Sigma-Aldrich).

Sequence analysis. Sequences were retrieved from The Arabidopsis Information Resource (TAIR) ${ }^{42}$ or GenBank ${ }^{43}$. Protein sequences were aligned using the ClustalW option with default parameters from Molecular Evolutionary Genetics Analysis (MEGA) version 6.0 (ref. 44). Phylogenetic trees created using the neighbour-joining statistical method and applying the bootstrap method with 1,000 replications and finally visualized using MEGA 6.

Heterologous expression and transporter assays. Heterologous expression was undertaken using the uracil-auxotrophic Saccharomyces cerevisiae (strain INVSc1: MATa his3D1 leu2 trp1-289 ura3-52 MAT his3D1 leu2 trp1-289 ura3-52, Thermo Fisher Scientific). Microsomal membranes were isolated from $500-\mathrm{ml}$ cultures grown at $30^{\circ} \mathrm{C}$ under constant shaking. The yeast pellet was resuspended in $10 \mathrm{ml}$ resuspension buffer $(50 \mathrm{mM}$ potassium phosphate, $\mathrm{pH} 7.1,1.4 \mathrm{M}$ sorbitol, $10 \mathrm{mM}$ $\mathrm{NaN}_{3}$ and $40 \mathrm{mM}$ 2-mercaptoethanol). To this, 6,000 units Lyticase (Sigma-Aldrich) was added and cells incubated for $1 \mathrm{~h}$ at $37^{\circ} \mathrm{C}$. Resultant spheroplasts were collected at $2,500 \mathrm{~g}$ for $5 \mathrm{~min}$, washed once with $0.8 \mathrm{M}$ sorbitol, $10 \mathrm{mM}$ triethanolamine/acetic acid (pH 7.2), $1 \mathrm{mM}$ EDTA and then lysed using acid-washed glass beads (Sigma-Aldrich) in $5 \mathrm{ml} 0.8 \mathrm{M}$ sorbitol, $10 \mathrm{mM}$ triethanolamine/acetic acid ( $\mathrm{pH} 7.2$ ) and $1 \mathrm{mM}$ EDTA, supplemented with a protease inhibitor cocktail (Sigma-Aldrich) and $1 \mathrm{mM}$ PMSF. The microsomal fraction was obtained by sequential centrifugation $(8,000 \mathrm{~g}$ for $10 \mathrm{~min}$ (F1), and 
$100,000 \mathrm{~g}$ for $75 \mathrm{~min}(\mathrm{~F} 2))$. The F2 fractions were resuspended in reconstitution buffer (10 mM Tricine- $\mathrm{KOH}$ ( $\mathrm{pH} 7.5$ ), $50 \mathrm{mM}$ potassium gluconate and $20 \%$ glycerol). Reconstitution of yeast microsomal proteins into liposomes was undertaken by the detergent solubilization and rapid removal approach. Approximately $600 \mathrm{mg}$ of acetone washed soybean L- $\alpha$-phosphatidylcholine (Avanti Polar Lipids) was dissolved in $10 \mathrm{ml}$ chloroform and evaporated under vacuum at $42^{\circ} \mathrm{C}$ for $45 \mathrm{~min}$. The resultant film was suspended in reconstitution buffer (10 mM Tricine- $\mathrm{KOH}$ (pH 7.5), $50 \mathrm{mM}$ potassium gluconate and $20 \%$ glycerol). Microsomal reconstitution was undertaken using around $400 \mu \mathrm{g}$ microsomal protein in reconstitution buffer, lipid at a ratio of 13 (lipid:protein), $10 \mathrm{mM}$ exchange substrate and $50 \mathrm{mM}$ octyl- $\beta$-glucoside. Unincorporated components were removed from reconstituted liposomes using Sephadex G50 (GE Healthcare). Aliquots of $200 \mu \mathrm{l}$ were incubated with nucleotide sugar substrates at $25^{\circ} \mathrm{C}$ for indicated times to assess transporter activities. Kinetic parameters were calculated by non-linear regression using the Prism 6 application (GraphPad Sofware, La Jolla, CA). Polyacrylamide gel electrophoreses was undertaken using $2.5 \mu \mathrm{g}$ protein of proteo-liposomes separated on a $7-15 \%$ SDS-polyacrylamide gel electrophoresis gel. The resolved proteins were transferred onto nitrocellulose (Hybond ECL, GE Healthcare), and immunoblotting was conducted with the anti-V5 antibody using a dilution of 1:10,000 (\#R96025, Thermo Fisher Scientific). Detection was accomplished with an anti-mouse IgG-peroxidase secondary antibody (\#A4416, Sigma-Aldrich) at a dilution of 1:20,000 in conjunction with the ECL Prime western blotting detection reagent (GE Healthcare) and a ChemiDoc Imaging Systems (Bio-Rad).

\section{Protein quantification by multiple reaction monitoring. Heterologous} expression in yeast was undertaken using the pYES-DEST52 vector, which contains an in-frame V5-tag. Absolute quantification of expressed proteins in yeast proteo-liposomes was undertaken by multiple reaction monitoring (MRM) analysis of a common tryptic peptide (R.SRGPFEGKPIPNPLLGLDSTR.T) to the V5-tag region using a 6460 Triple Quad LC/MS system (Agilent Technologies, CA). A synthetic peptide was used to determine the following parameters for MRM: Dwell $(25 \mathrm{msec})$, Fragmentor (130 V), Collision Energy (11.1 V) and Cell Accelerator Voltage (7 V). Proteo-liposomes $(50 \mu \mathrm{g})$ containing expressed NSTs were digested with trypsin $(5 \mu \mathrm{g})$ in $25 \mathrm{mM}$ ammonium carbonate and $50 \%$ methanol (v/v), and incubated overnight at $37^{\circ} \mathrm{C}$. Resultant peptides were concentrated and resuspended in water containing $0.1 \%$ formic acid before analysis by LC-MS/MS. The system was operated in positive ion mode using the MRM scan type with both MS1 and MS2 set to Unit resolution and the following parameters: gas temperature $\left(350^{\circ} \mathrm{C}\right)$, gas flow $\left(10 \mathrm{lmin}^{-1}\right)$, nebulizer $(45$ p.s.i.), sheath gas temperature $\left(400^{\circ} \mathrm{C}\right)$, sheath gas flow $\left(11 \mathrm{lmin}^{-1}\right)$, capillary $(5,000 \mathrm{~V})$ and MS1/MS2 heater $\left(100^{\circ} \mathrm{C}\right)$. A total of $10 \mu \mathrm{g}$ of peptides was loaded onto a Ascentis Express Peptide ES-C18 $(5 \mathrm{~cm} \times 2.1 \mathrm{~mm}, 2.7 \mu \mathrm{m})$ column (Sigma-Aldrich) using a 1290 series HPLC (Agilent Technologies) at a flow rate of $0.4 \mathrm{ml} \mathrm{min}^{-1}$ as follows: $95 \%$ buffer A ( $99.9 \%$ water and $0.1 \%$ formic acid) and 5\% buffer B $(99.9 \%$ acetonitrile and $0.1 \%$ formic acid) for $0.2 \mathrm{~min}$, followed by an increase to $35 \%$ buffer B over $5.5 \mathrm{~min}$, then $90 \%$ buffer B in $0.3 \mathrm{~min}$, where it was held for $2 \mathrm{~min}$ and back to $5 \%$ buffer $\mathrm{B}$ over $5 \mathrm{~min}$, giving total runtime of $13 \mathrm{~min}$. The column temperature was maintained at $60^{\circ} \mathrm{C}$. Data were acquired using MassHunter Workstation Software Version B.06.00 Build 6.0.6025.4 SP4 (Agilent Technologies). The synthetic peptide standard was used to create a standard curve by linear regression using a range of abundances $(0.5-10 \mathrm{pmol})$. Resultant data were imported into Skyline (v2.5.0.6157) ${ }^{45}$ and peaks manually inspected for retention time and adjusted accordingly. The abundance of an expressed protein in the sample $(10 \mu \mathrm{g})$ was calculated by integrating the total signal peak area (total area) from Skyline for two transitions on the $563.560[\mathrm{M}+4 \mathrm{H}]^{4+}$ precursor ion, namely, $\mathrm{L}[y 7] 761.452[\mathrm{M}+\mathrm{H}]^{1+}$ and $\mathrm{G}[y 6] 648.3311[\mathrm{M}+\mathrm{H}]^{1+}$ and calculating total moles in the sample against the standard curve. The expressed NSTs comprise $\sim 0.5 \%$ of total protein in a standard proteo-liposome preparation (Supplementary Table 1). These values were used for the kinetic calculations outlined in Table 1.

Plant growth and transformation. Arabidopsis thaliana (L.) Heynh. Columbia-0 (Col-0) was obtained from the Arabidopsis Biological Resource Center (http:// abrc.osu.edu/). The T-DNA insertion mutants for GFT1 (SALK 094857; SAIL_400_E04) were obtained from the SIGnAL Salk collection ${ }^{46}$ and Syngenta Arabidopsis Insertion Library (SAIL) collection ${ }^{47}$. A hairpin construct specifically targeting GFT1 expression was assembled using the pHANNIBAL/pART27 system $^{48}$ and designated hpGFT1. A 506-bp fragment was amplified from genomic DNA using the following primer pairs: hpGFT1s_fwd (5'-acggaattccgAAGTCGT CGCCTTCCTCTAATT- $\left.3^{\prime}\right)$, hpGFT1s_rev ( $5^{\prime}$-ggggtaccccTCGAAGGTAAAGGC TGACTACGA- $\left.3^{\prime}\right)$ and hpGFTlas_fwd (5' -agctctagagcAAGTCGTCGCCTTCC TCTAATT- $\left.3^{\prime}\right)$, hpGFT1as_rev ( $5^{\prime}$-ccatcgatggTCGAAGGTAAAGGCTGACTA CGA- $3^{\prime}$ ), and cloned into the pHANNIBAL vector in sense and antisense orientation at the EcoRI to Asp718 and XbaI to ClaI restriction sites. The expression cassette was subsequently excised from the pHANNIBAL vector using NotI and sub-cloned into the corresponding Not1 site of the plant transformation vector pART27. For kanamycin selection, seeds were germinated on halfconcentrated MS medium ${ }^{49}$ supplemented with $1 \%(\mathrm{w} / \mathrm{v})$ sucrose, $75 \mu \mathrm{g} \mathrm{ml} \mathrm{g}^{-1}$ kanamycin, $100 \mu \mathrm{g} \mathrm{ml}^{-1}$ Cefotaxime (Sigma-Aldrich) and solidified with $0.7 \%$ (w/v) agar under a $16-\mathrm{h}$ photoperiod $\left(120 \mu \mathrm{mol} \mathrm{m}^{-2} \mathrm{~s}^{-1}, 22^{\circ} \mathrm{C}\right)$. Plants were transferred to soil (PRO-MIX, Premier Horticulture Inc.) and further grown in an Arabidopsis growth chamber (Percival-Scientific) under short-day light conditions ( $10 \mathrm{~h}$ of fluorescent light $\left(120 \mu \mathrm{mol} \mathrm{m}^{-2} \mathrm{~s}^{-1}\right)$ at $22^{\circ} \mathrm{C}$ and $60 \%$ relative humidity (RH) $/ 14 \mathrm{~h}$ of dark at $22^{\circ} \mathrm{C}$ and $\left.60 \% \mathrm{RH}\right)$. After 4 weeks plants were transferred to long-day conditions $\left(16 \mathrm{~h}\right.$ of fluorescent light $\left(120 \mu \mathrm{mol} \mathrm{m}^{-2} \mathrm{~s}^{-1}\right)$ at $22^{\circ} \mathrm{C}$ and $60 \% \mathrm{RH} / 8 \mathrm{~h}$ of dark at $22^{\circ} \mathrm{C}$ and $\left.60 \% \mathrm{RH}\right)$.

Cloning procedures. Coding sequences for Arabidopsis GONST3 and GFT1 without the native stop codon were PCR amplified from genomic DNA and GONST1 was PCR amplified from leaf cDNA using the following primer pairs: GONST3-fwd (5'-CACCATGTCGACGAATGATGAGGAAAATGG-3'), GONST3-rev (5'-TAGTTTCTCTTCTGATTTCAGAGTTTCCTT-3'); GFT1-fwd (5'- CACCATGTCGTCCTCTCGATTCGATTCAA-3'), GFT1-rev (5' GAAGCTAGTTTCCCCGG-3 ${ }^{\prime}$ ); and GONST1-fwd ( $5^{\prime}$-CACCATGAAATTGTAC GAACACGATGGAGTTGA-3'), GONST1-rev (5'-GGACTTCTCCCTCATTT TGGCTCTAGCA-3'). GONST2 was synthesized according the current gene model (TAIR10; GenScript, Piscataway, NJ) and amplified using the following primer pair GONST2-fwd (5'-CACCATGTCTGCCGTGAAACTGGAAGC-3') and GONST2-rev ( $5^{\prime}$-TGACATTTTAGCTCTGGCAAAGACCACT- $3^{\prime}$ ). To generate the respective entry clones, the resultant PCR products were introduced into the pENTR/SD/D-TOPO cloning vector (Life Technologies) according to the instructions of the manufacturer. C-terminal YFP fusions were created by introduction of the constructs into the $35 \mathrm{~S}$ promoter carrying pEarleyGate101 plant transformation vector ${ }^{50}$, using the LR Clonase II reaction (Life Technologies) following the manufacturer's protocol. To obtain yeast expression clones, the constructs were subsequently cloned into the yeast expression vector pYES-DEST52 (Life Technologies) using LR Clonase II (Life Technologies).

Sub-cellular localization and microscopy. For transient protein expression in $N$. benthamiana (tobacco) leaves, Agrobacterium tumefaciens strain GV3101 pmp90 carrying the C-terminal YFP fusion constructs and the aMan-mCherry Golgi marker (G-rk) or the mCherry ER marker (ER-rk) ${ }^{51}$ were grown overnight in Luria-Bertani (LB) media. After a centrifugation step $\left(10 \mathrm{~min}, 4,000 \mathrm{~g}, 15^{\circ} \mathrm{C}\right)$, the supernatant was discarded and the cell pellets were resuspended in infiltration buffer containing $10 \mathrm{mM}$ MES, $10 \mathrm{mM} \mathrm{MgCl}$ and $100 \mu \mathrm{M}$ acetosyringone to an $\mathrm{OD}_{600}=0.01-0.15$. Subsequently, leaves of 4 -week-old $N$. benthamina plants (grown at $25 / 24^{\circ} \mathrm{C}$ day/night temperature, $60 \%$ humidity and 16 -h light $/ 8$-h dark cycles) were co-infiltrated with the Agrobacterium mixtures in infiltration buffer using a 1-ml syringe. After infiltration, plants were moved back to the growth room and protein expression was monitored 2 days post infiltration. Visualization by confocal laser scanning microscopy was performed using excitation wavelengths of $514 \mathrm{~nm}$ (YFP) and $580 \mathrm{~nm}$ (mCherry). Emissions were collected at $520-560 \mathrm{~nm}$ (YFP) and 600-630 nm (mCherry). The pinhole diameter was set at 1 Airy unit and a $\times 631.4$ numerical aperture oil immersion objective was used. Raw images were processed using Image ${ }^{52}$. Background signals were subtracted using negative control images taken from the same experimental material. The Co-localization threshold tool in ImageJ was used to calculate the Manders' tM1 and tM2 overlap coefficients on regions of interest to quantify co-localization between the fluorescently labelled proteins.

Categorization of hpGFT1 plants. The 4-week-old T1 hpGFT1 plants were divided into four cohorts based on the rosette size when compared with the vector control lines. The hpGFT1 plants with rosettes $>50 \%$ of control lines were assigned as cohort \#1, plants with rosettes $\sim 50 \%$ were assigned as cohort \#2, plants with rosettes $\sim 25 \%$ were assigned as cohort \#3 and plants with rosettes $<25 \%$ were assigned as cohort \#4. Due to limitations in plant tissue from cohorts \#3 and \#4, plant material for all downstream analyses was derived from 5 to10 pooled individuals for all lines.

Cell wall monosaccharide composition. Plant material frozen in liquid nitrogen was ground to a fine powder before boiling in $96 \%$ ethanol for $30 \mathrm{~min}$. After a centrifugation step of $5 \mathrm{~min}$ at $20,000 \mathrm{~g}$, the supernatant was discarded. The resultant pellet was washed with $70 \%$ ethanol until chlorophyll was removed. Last, the pellet was washed with $100 \%$ acetone and dried in a vacuum concentrator. Samples were hydrolysed in $2 \mathrm{~N}$ trifluoroacetic acid (TFA) for $1 \mathrm{~h}$ at $120^{\circ} \mathrm{C}$. High-performance anion exchange chromatography with pulsed amperometric detection was performed on an ICS 3000 (Dionex Corporation, Sunnyvale, CA) using a CarboPac PA20 $(3 \times 150 \mathrm{~mm}$, Dionex Corporation, Sunnyvale, CA $)$ anion exchange column at a flow rate of $0.4 \mathrm{ml} \mathrm{min}^{-1}$. Before sample injection, the column was equilibrated with $10 \mathrm{mM} \mathrm{NaOH}$ for $10 \mathrm{~min}$. The elution program involved two isocratic elution steps with $10 \mathrm{mM} \mathrm{NaOH}$ from 0 to $15 \mathrm{~min}$ to separate the neutral sugars followed by a ramp step to $450 \mathrm{mM} \mathrm{NaOH}$ from 15.1 to $35 \mathrm{~min}$, which allowed separation of uronic acids and washing of the column. Monosaccharide standards comprised L-Fuc, L-Rha, L-Ara, CGal, D-Glc, D-Xyl, $\mathrm{D}$-GalA and D-GlcA. A run of a standard mixture containing the eight monosaccharides was performed with each sample set to enable sample quantitation by linear regression. 
Mutant identification by PCR. To identify homozygous T-DNA insertion lines, PCR was performed using the following primers: SAIL_400_E04 (5' ${ }^{\prime}$-TTCCATTA ATGGTGAGTTGACC-3') (fwd) and (5'-CTGCTGATAACCAACACCTCC- $\left.3^{\prime}\right)$ (rev), and SALK_094857 with ( $5^{\prime}$-AGCGTTGGTTCAGCACATATC- $\left.3^{\prime}\right)$ (fwd) and (5'-AGGAAACCAAACACACACGAC-3') (rev). For the PCR verifying the T-DNA, the SAIL_LB ( $5^{\prime}$-GCCTTTTCAGAAATGGATAAATAGCCTTGCT TCC- ${ }^{\prime}$ ) and the SALK_LBb1.3 (5'-ATTTTGCCGATTTCGGAAC-3') were used.

Quantitative PCR. RNA was extracted from plant tissue using the RNeasy RNA Plant kit (Qiagen) and a total of $0.5-1 \mu \mathrm{g}$ was reverse-transcribed using SuperScriptII reverse transcriptase (Thermo Fisher Scientific) and $\mathrm{d}(\mathrm{T})_{15}$ oligomers (Life Technologies). To determine GFT1 and GONST3 expression in hpGFT1 lines, resultant cDNA was subsequently used as template in a reverse transcription qPCR reaction containing $2 \times$ SYBR Select Master Mix (Applied Biosystems) and genespecific primers. GFT1 was amplified with (5'-GCGTCAACTCCTCATCTTCC- $\left.3^{\prime}\right)$ (fwd) and (5'-TAAGCATGCCACTCCTGTTG-3') (rev), and GONST3 with (5'-GGAAACGCTTTTCTTGCATC-3') (fwd) and (5'-ACGCAAGAGCCCAGC TATAA- $3^{\prime}$ ) (rev). As a reference, the Arabidopsis ubiquitin-10 gene (At4g05320) was amplified using the primers ( $5^{\prime}$-GGCCTTGTATAATCCCTGATGAAT AAG- $\left.3^{\prime}\right)$ (fwd) and $\left(5^{\prime}\right.$-AAAGAGATAACAGGAACGGAAACATAGT- $\left.3^{\prime}\right)$ (rev), and the Arabidopsis PP2A gene (Atlg13320) was amplified using the primers (5'-TAACGTGGCCAAAATGATGC- ${ }^{\prime}$ ) (fwd) and (5'-GTTCTCCACAACCGC TTGGT- $3^{\prime}$ ) (rev). The PCR reactions were performed using the StepOnePlus Real-Time PCR (Applied Biosystems) system and the following thermal profile was used for all PCR reactions: $50^{\circ} \mathrm{C}$ for $2 \mathrm{~min}, 95^{\circ} \mathrm{C}$ for $10 \mathrm{~min}, 40$ cycles of $95^{\circ} \mathrm{C}$ for $15 \mathrm{~s}$ and $60^{\circ} \mathrm{C}$ for $1 \mathrm{~min}$. Amplicon dissociation curves (melting curves) were recorded after cycle 40 by heating from 60 to $95^{\circ} \mathrm{C}$ with a ramp speed of $1.9^{\circ} \mathrm{C} \mathrm{min}{ }^{-1}$. Data were analysed using the SDS 2.2.1 software (Applied Biosystems). Relative expression levels were determined using the geNorm algorithm ${ }^{53}$, which uses the geometric mean of the housekeeping genes (UBI10 and PP2A).

Protein extraction and immunoblotting. Protein was extracted from plant material by grinding in extraction buffer $(10 \mathrm{mM}$ Tris ( $\mathrm{pH} 8), 150 \mathrm{mM} \mathrm{NaCl}, 2 \%$ Triton, $1 \mathrm{mM}$ PMSF, protease inhibitor and $10 \mathrm{mM} \mathrm{CaCl}_{2}$ ) and incubated for $1 \mathrm{~h}$ at $4{ }^{\circ} \mathrm{C}$ under constant shaking. The homogenate was centrifuged for $30 \mathrm{~min}$ at $20,000 \mathrm{~g}$ at $4{ }^{\circ} \mathrm{C}$ to remove cell debris. Proteins were precipitated with $20 \%$ trichloroacetic acid after incubation on ice and centrifuged at $20,000 \mathrm{~g}$ at $4{ }^{\circ} \mathrm{C}$ After removal of the supernatant, samples were washed twice with ice-cold acetone and dried using a vacuum concentrator. For immunoblotting, proteins were suspended in SDS sample buffer and separated by SDS-polyacrylamide gel electrophoresis (7-15\%) and transferred onto nitrocellulose (Hybond ECL, GE Healthcare). $N$-glycan epitopes were detected using antibodies raised against $\beta-(1,2)$-xylose (\#AS07 267) and $\alpha$-(1,3)-fucose (\#AS07 268; Agrisera, Sweden) using dilutions1:10,000 and 1:5,000, respectively. Detection was accomplished with an anti-rabbit IgG-peroxidase secondary antibody (\#A9169, Sigma-Aldrich) at a dilution of 1:20,000 in conjunction with the ECL Prime western blotting detection reagent (GE Healthcare) and a ChemiDoc Imaging System (Bio-Rad).

Oligosaccharide mass profiling. Plant tissue was frozen in liquid nitrogen and homogenized using a mixer mill (Retsch), resuspended in 96\% ethanol, and incubated at $100^{\circ} \mathrm{C}$ for $30 \mathrm{~min}$. The pellet was then collected by centrifugation $(20,000 \mathrm{~g}$ for $5 \mathrm{~min})$ and subjected to three wash steps: two washes with $70 \%$ ethanol, followed by a wash with $100 \%$ acetone. The remaining pellet was dried in a vacuum concentrator. Samples were digested with a xyloglucan-specific endoglucanase from Aspergillus aculeatus ${ }^{36}$ in $50 \mathrm{mM}$ ammonium formate ( $\mathrm{pH} 4.5$ ), for $16 \mathrm{~h}$ at $37^{\circ} \mathrm{C}$. Following digestion, the solubilized xyloglucan oligosaccharides were collected by centrifugation $(1,000 \mathrm{~g}$ for $5 \mathrm{~min})$ and desalted using the BioRex MSZ 501 cation exchange resin beads (Bio-Rad). A total of $2 \mu \mathrm{l}$ of the desalted sample was spotted onto a MALDI plate with 2,5-dihydroxybenzoic acid and analysed using an AXIMA (Shimadzu Corporation) matrix-assisted laser desorption/ionization time-of-flight set in linear positive mode with an acceleration voltage of $20,000 \mathrm{~V}$.

Extraction of nucleotide sugars. Frozen plant material $(\sim 40 \mathrm{mg})$ was homogenized using a mixer mill (Retsch) and $600 \mu \mathrm{l}$ of ice-cold chloroform/methanol solution $(30 \%$ chloroform $/ 70 \%$ methanol) added to each sample. The homogenate was incubated at $-20^{\circ} \mathrm{C}$ for $2 \mathrm{~h}$, then $400 \mu \mathrm{l}$ ice-cold water was added and samples were mixed and centrifuged at $20,000 \mathrm{~g}$ for $5 \mathrm{~min}$ at $4{ }^{\circ} \mathrm{C}$. The upper phase was transferred to a $15-\mathrm{ml}$ tube. This step was repeated twice and the combined upper phases were lyophilized overnight. The dried extracts were dissolved in $1 \mathrm{ml} 10 \mathrm{mM}$ ammonium bicarbonate before purification using ENVI-Carb SPE columns (Sigma-Aldrich). Eluates were lyophilized overnight, resuspended in ice-cold water and analysed by LC-MS/MS as described below.

Analysis of nucleotide sugars by tandem mass spectrometry. Tandem mass spectrometry (LC-MS/MS) was performed at a flow rate of $50 \mu \mathrm{lmin}{ }^{-1}$ using a Hypercarb column $(150 \mathrm{~mm} \times 1 \mathrm{~mm}, 5 \mu \mathrm{m})$, as the stationary phase with an 1100 series HPLC system (Agilent Technologies) and a 4000 QTRAP LC-MS/MS system (Sciex, CA) equipped with a TurbolonSpray ion source. The system was run in Micro mode with a mix rate of $400 \mu \mathrm{min}^{-1}$ and the column compartment was set to $50^{\circ} \mathrm{C}$ with samples kept at $4{ }^{\circ} \mathrm{C}$. Initial conditions were $95 \%$ buffer A (LC-MS grade water with $0.3 \%$ formic acid, $\mathrm{pH} 9.0$ with ammonia) and $5 \%$ buffer B $(100 \%$ acetonitrile) for $1 \mathrm{~min}$ followed by a gradient to $75 \%$ (A) in $20 \mathrm{~min}$, then $50 \%$ (A) in $5 \mathrm{~min}$ before returning to $95 \%$ (A) in $5 \mathrm{~min}$. Specific compound-dependent MS parameters for each nucleotide sugar were determined previously ${ }^{20}$. The 4000 QTRAP was operated in negative ion mode using the MRM scan type. A declustering potential of -40 , entrance potential of -10 , collision cell exit potential was -15 . The ion spray voltage was set at $-4,200 \mathrm{~V}$, source temperature (TEM) at $425^{\circ} \mathrm{C}$, collision gas (CAD) was set to high and source gas 1 (GS1) and 2 (GS2) were both set to 20 . A time of $100 \mathrm{~ms}$ was applied for each transition resulting in a duty cycle of $1.0501 \mathrm{~s}$ with both Q1 and Q3 resolutions set to Unit. All data were acquired using Analyst 1.5.1 Build 5218 (Sciex, CA) operating in MRM mode. Nucleotide sugars were quantified using MultiQuant 2.1 (build 2.1.1296.02.1) software (Sciex, CA) by integrating the signal peak areas

Data availability. The nucleotide sequences for cloned Arabidopsis GONST family members have been deposited in the GenBank with accession codes KR265320 (At2g13650, GONST1), KR265321 (At1g07290, GONST2) and KR265322 (At5g19980, GFT1). All other data supporting the findings of this study are available within the article and its Supplementary Information files or from the corresponding author on request.

\section{References}

1. Bar-Peled, M. \& O’Neill, M. A. Plant nucleotide sugar formation, interconversion, and salvage by sugar recycling. Annu. Rev. Plant Biol. 62, 127-155 (2011).

2. Mortimer, J. C. et al. Abnormal glycosphingolipid mannosylation triggers salicylic acid-mediated responses in Arabidopsis. Plant Cell 25, 1881-1894 (2013).

3. Jadid, N. et al. DOLICHOL PHOSPHATE MANNOSE SYNTHASE1 mediates the biogenesis of isoprenyl-linked glycans and influences development, stress response, and ammonium hypersensitivity in Arabidopsis. Plant Cell 23, 1985-2005 (2011).

4. Zhang, M. et al. LEW3, encoding a putative alpha-1,2-mannosyltransferase (ALG11) in N-linked glycoprotein, plays vital roles in cell-wall biosynthesis and the abiotic stress response in Arabidopsis thaliana. Plant J. 60, 983-999 (2009).

5. Liepman, A. H., Wilkerson, C. G. \& Keegstra, K. Expression of cellulose synthase-like $(C s l)$ genes in insect cells reveals that $C s l A$ family members encode mannan synthases. Proc. Natl Acad. Sci. USA 102, 2221-2226 (2005).

6. Vanzin, G. F. et al. The mur2 mutant of Arabidopsis thaliana lacks fucosylated xyloglucan because of a lesion in fucosyltransferase AtFUT1. Proc. Natl Acad. Sci. USA 99, 3340-3345 (2002)

7. Strasser, R., Altmann, F., Mach, L., Glossl, J. \& Steinkellner, H. Generation of Arabidopsis thaliana plants with complex $N$-glycans lacking beta 1,2 -linked xylose and core alpha 1,3-linked fucose. FEBS Lett. 561, 132-136 (2004).

8. Liang, Y. et al. Biochemical and physiological characterization of fut 4 and fut6 mutants defective in arabinogalactan-protein fucosylation in Arabidopsis. J. Exp. Bot. 64, 5537-5551 (2013).

9. Pabst, M. et al. Rhamnogalacturonan II structure shows variation in the side chains monosaccharide composition and methylation status within and across different plant species. Plant J. 76, 61-72 (2013).

10. Bonin, C. P. \& Reiter, W. D. A bifunctional epimerase-reductase acts downstream of the MUR1 gene product and completes the de novo synthesis of GDP-L-fucose in Arabidopsis. Plant J. 21, 445-454 (2000).

11. Bonin, C. P., Potter, I., Vanzin, G. F. \& Reiter, W. D. The MUR1 gene of Arabidopsis thaliana encodes an isoform of GDP-D-mannose-4,6-dehydratase, catalyzing the first step in the de novo synthesis of GDP-L-fucose. Proc. Natl Acad. Sci. USA 94, 2085-2090 (1997).

12. Zablackis, E. et al. Substitution of L-fucose by L-galactose in cell walls of Arabidopsis mur1. Science 272, 1808-1810 (1996).

13. Pauly, M., Eberhard, S., Albersheim, P., Darvill, A. \& York, W. S. Effects of the mur1 mutation on xyloglucans produced by suspension-cultured Arabidopsis thaliana cells. Planta 214, 67-74 (2001).

14. Rayon, C. et al. Characterization of $N$-glycans from Arabidopsis. Application to a fucose-deficient mutant. Plant Physiol. 119, 725-733 (1999).

15. Reuhs, B. L. et al. L-Galactose replaces L-fucose in the pectic polysaccharide rhamnogalacturonan II synthesized by the L-fucose-deficient mur1 Arabidopsis mutant. Planta 219, 147-157 (2004).

16. Tryfona, T. et al. Characterisation of FUT4 and FUT6 $\alpha$-(1->2)fucosyltransferases reveals that absence of root arabinogalactan fucosylation increases Arabidopsis root growth salt sensitivity. PLOS ONE 9, e93291 (2014).

17. Rollwitz, I., Santaella, M., Hille, D., Flugge, U. I. \& Fischer, K. Characterization of AtNST-KT1, a novel UDP-galactose transporter from Arabidopsis thaliana. FEBS Lett. 580, 4246-4251 (2006). 
18. Reyes, F. \& Orellana, A. Golgi transporters: opening the gate to cell wall polysaccharide biosynthesis. Curr. Opin. Plant Biol. 11, 244-251 (2008).

19. Seino, J. et al. Characterization of rice nucleotide sugar transporters capable of transporting UDP-galactose and UDP-glucose. J. Biochem. 148, 35-46 (2010).

20. Rautengarten, C. et al. The Golgi localized bifunctional UDP-rhamnose/UDPgalactose transporter family of Arabidopsis. Proc. Natl Acad. Sci. USA 111, 11563-11568 (2014).

21. Ebert, B. et al. Identification and characterization of a Golgi localized UDPxylose transporter family from Arabidopsis. Plant Cell 27, 1218-1227 (2015).

22. Niemann, M. C. E. et al. Arabidopsis ROCK1 transports UDP-GlcNAc/UDPGalNAc and regulates ER protein quality control and cytokinin activity. Proc. Natl Acad. Sci. USA 112, 291-296 (2015).

23. Baldwin, T. C., Handford, M. G., Yuseff, M. I., Orellana, A. \& Dupree, P. Identification and characterization of GONST1, a Golgi-localized GDPmannose transporter in Arabidopsis. Plant Cell 13, 2283-2295 (2001).

24. Handford, M. G., Sicilia, F., Brandizzi, F., Chung, J. H. \& Dupree, P. Arabidopsis thaliana expresses multiple Golgi-localised nucleotide-sugar transporters related to GONST1. Mol. Genet. Genomics 272, 397-410 (2004).

25. Wulff, C., Norambuena, L. \& Orellana, A. GDP-fucose uptake into the Golgi apparatus during xyloglucan biosynthesis requires the activity of a transporter-like protein other than the UDP-glucose transporter. Plant Physiol. 122, 867-877 (2000).

26. Dean, N., Zhang, Y. B. \& Poster, J. B. The VRG4 gene is required for GDP-mannose transport into the lumen of the Golgi in the yeast Saccharomyces cerevisiae. J. Biol. Chem. 272, 31908-31914 (1997).

27. Handford, M. et al. Arabidopsis thaliana AtUTr7 encodes a golgi-localized UDP-glucose/UDP-galactose transporter that affects lateral root emergence. Mol. Plant 5, 1263-1280 (2012).

28. Schmid, M. et al. A gene expression map of Arabidopsis thaliana development. Nat. Genet. 37, 501-506 (2005).

29. Parsons, H. T. et al. Isolation and proteomic characterization of the Arabidopsis Golgi defines functional and novel components involved in plant cell wall biosynthesis. Plant Physiol. 159, 12-26 (2012).

30. Colleoni, C. et al. Phylogenetic and biochemical evidence supports the recruitment of an ADP-glucose translocator for the export of photosynthate during plastid endosymbiosis. Mol. Biol. Evol. 27, 2691-2701 (2010).

31. Fry, S. C. et al. An unambiguous nomenclature for xyloglucan-derived oligosaccharides. Physiol. Plantarum 89, 1-3 (1993).

32. Davis, J., Brandizzi, F., Liepman, A. H. \& Keegstra, K. Arabidopsis mannan synthase CSLA9 and glucan synthase CSLC4 have opposite orientations in the Golgi membrane. Plant J. 64, 1028-1037 (2010).

33. Utz, D. \& Handford, M. VvGONST-A and VvGONST-B are Golgi-localised GDP-sugar transporters in grapevine (Vitis vinifera L.). Plant Sci. 231, 191-197 (2015).

34. Luhn, K., Wild, M. K., Eckhardt, M., Gerardy-Schahn, R. \& Vestweber, D. The gene defective in leukocyte adhesion deficiency II encodes a putative GDP-fucose transporter. Nat. Genet. 28, 69-72 (2001).

35. O’Neill, M. A., Eberhard, S., Albersheim, P. \& Darvill, A. G. Requirement of borate cross-linking of cell wall rhamnogalacturonan II for Arabidopsis growth. Science 294, 846-849 (2001).

36. Pauly, M. et al. A xyloglucan-specific endo- $\beta$-1,4-glucanase from Aspergillus aculeatus: expression cloning in yeast, purification and characterization of the recombinant enzyme. Glycobiology 9, 93-100 (1999).

37. Kang, J. S. et al. Salt tolerance of Arabidopsis thaliana requires maturation of $N$-glycosylated proteins in the Golgi apparatus. Proc. Natl Acad. Sci. USA 105, 5933-5938 (2008)

38. Kaulfürst-Soboll, H. et al. Reduced immunogenicity of Arabidopsis hgll mutant $N$-glycans caused by altered accessibility of xylose and core fucose epitopes. J. Biol. Chem. 286, 22955-22964 (2011).

39. Bonin, C. P., Freshour, G., Hahn, M. G., Vanzin, G. F. \& Reiter, W. D. The GMD1 and GMD2 genes of Arabidopsis encode Isoforms of GDP-D-mannose 4,6-dehydratase with cell type-specific expression patterns. Plant Physiol. 132, 883-892 (2003).

40. Major, L. L., Wolucka, B. A. \& Naismith, J. H. Structure and function of GDP-mannose- $3^{\prime}, 5^{\prime}$-epimerase: an enzyme which performs three chemical reactions at the same active site. J. Am. Chem. Soc. 127, 18309-18320 (2005).

41. Rautengarten, C. et al. The interconversion of UDP-arabinopyranose and UDP-arabinofuranose is indispensable for plant development in Arabidopsis. Plant Cell 23, 1373-1390 (2011).
42. Lamesch, P. et al. The Arabidopsis Information Resource (TAIR): improved gene annotation and new tools. Nucleic Acids Res. 40, D1202-D1210 (2012).

43. Benson, D. A., Karsch-Mizrachi, I., Lipman, D. J., Ostell, J. \& Sayers, E. W. GenBank. Nucleic Acids Res. 39, D32-D37 (2011).

44. Tamura, K., Stecher, G., Peterson, D., Filipski, A. \& Kumar, S. MEGA6: Molecular Evolutionary Genetics Analysis version 6.0. Mol. Biol. Evol. 30, 2725-2729 (2013).

45. MacLean, B. et al. Skyline: an open source document editor for creating and analyzing targeted proteomics experiments. Bioinformatics 26, 966-968 (2010)

46. Alonso, J. M. et al. Genome-wide Insertional mutagenesis of Arabidopsis thaliana. Science 301, 653-657 (2003).

47. Sessions, A. et al. A high-throughput Arabidopsis reverse genetics system. Plant Cell 14, 2985-2994 (2002).

48. Wesley, S. V. et al. Construct design for efficient, effective and high-throughput gene silencing in plants. Plant J. 27, 581-590 (2001).

49. Murashige, T. \& Skoog, F. A revised medium for rapid growth and bio assays with tobacco tissue cultures. Physiol. Plantarum 15, 473-497 (1962).

50. Earley, K. W. et al. Gateway-compatible vectors for plant functional genomics and proteomics. Plant J. 45, 616-629 (2006).

51. Nelson, B. K., Cai, X. \& Nebenfuhr, A. A multicolored set of in vivo organelle markers for co-localization studies in Arabidopsis and other plants. Plant J. 51, 1126-1136 (2007).

52. Schneider, C. A., Rasband, W. S. \& Eliceiri, K. W. NIH Image to ImageJ: 25 years of image analysis. Nat. Methods 9, 671-675 (2012).

53. Vandesompele, J. et al. Accurate normalization of real-time quantitative RT-PCR data by geometric averaging of multiple internal control genes. Genome Biol. 3, research0034.1-0034.11 (2002).

\section{Acknowledgements}

This work was supported by the US Department of Energy, Office of Science, Office of Biological and Environmental Research, through contract DE-AC02-05CH11231 between Lawrence Berkeley National Laboratory and the US Department of Energy. J.L.H. is supported by an Australian Research Council Future Fellowship (FT130101165) Part of the work was supported by the Danish Strategic Research Council (Set4Future 11-116795). A.O. is supported by Fondecyt 1151335, FONDAP-CRG 15090007 and Basal Program PB-16. The substrates obtained from Carbosource Services (Athens, GA) were supported in part by NSF-RCN grant \#0090281. We also thank the Biological Optical Microscopy Platform (BOMP) at the University of Melbourne for technical support.

\section{Author contributions}

C.R., B.E., A.O., H.V.S. and J.L.H. designed the project and analysed the data. A.M.S.-M., J.L.H. and C.R. conducted the statistical analysis. C.R. and B.E. performed the experiments. S.S. performed sub-cellular localizations. L.L. and M.P. performed the OLIMP analysis. C.R., B.E. and J.L.H. wrote the manuscript.

\section{Additional information}

Supplementary Information accompanies this paper at http://www.nature.com/ naturecommunications

Competing financial interests: The authors declare no competing financial interests.

Reprints and permission information is available online at http://npg.nature.com/ reprintsandpermissions/

How to cite this article: Rautengarten, C. et al. The Arabidopsis Golgi-localized GDP-L-fucose transporter is required for plant development. Nat. Commun. 7:12119 doi: 10.1038/ncomms12119 (2016)

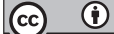

This work is licensed under a Creative Commons Attribution 4.0 International License. The images or other third party material in this article are included in the article's Creative Commons license, unless indicated otherwise in the credit line; if the material is not included under the Creative Commons license, users will need to obtain permission from the license holder to reproduce the material To view a copy of this license, visit http://creativecommons.org/licenses/by/4.0/

(C) The Author(s) 2016 